\title{
Nonlinear resultant theory of shells accounting for thermodiffusion
}

\author{
In occasion of the 65th birthday of Prof. Holm Altenbach
}

Received: 22 August 2020 / Accepted: 11 September 2020 / Published online: 28 September 2020

(C) The Author(s) 2020

\begin{abstract}
The complete nonlinear resultant 2D model of shell thermodiffusion is developed. All 2D balance laws and the entropy imbalance are formulated by direct through-the-thickness integration of respective 3D laws of continuum thermodiffusion. This leads to a more rich thermodynamic structure of our 2D model with several additional 2D fields not present in the 3D parent model. Constitutive equations of elastic thermodiffusive shells are discussed in more detail. They are formulated from restrictions imposed by the resultant 2D entropy imbalance according to Coleman-Noll procedure extended by a set of $2 \mathrm{D}$ constitutive equations based on heuristic assumptions.
\end{abstract}

Keywords Thermodiffusion · Nonlinear shell · Thermodynamics · Resultant shell theory

\section{Introduction}

Different approaches to description of thermodiffusion phenomena in 3D bodies were proposed in the literature, see the historic review by [38]. A deeper physical understanding of diffusion indicates that it is caused by Brownian motion of atoms and molecules, which can jump in large numbers between various interstitial sites in the body structure. Thus, most theories of diffusion in 3D bodies are based on the random walk theory and laws of statistical physics. They are used to model and analyse many processes in modern micro- and nanotechnology leading to development of such devices as computer chips, accumulator batteries, solid-state lasers, elements of mobile phones and so on. This approach is partly summarized, for example, by [18,37,41,53], where many references are available.

In the present paper, we are interested in developing the alternative nonlinear phenomenologic continuum model of thermodiffusion as applied to thin-walled solid shell structures. The first empirical law for the 3D diffusion flux of the species in the body was proposed already by [25] as the linear function of species concentration gradient field. Later experiments indicated that in various circumstances, diffusion coefficients in the Fick law may depend also on temperature, direction, internal stress, deformation and structure of the

Communicated by Andreas Öchsner.

V.A.E. acknowledges the support of the Government of the Russian Federation (contract No. 14.Z50.31.0046)

V. A. Eremeyev $(\varangle) \cdot$ W. Pietraszkiewicz

Faculty of Civil and Environmental Engineering, Gdańsk University of Technology, ul. Gabriela Narutowicza 11/12, 80-233 Gdańsk, Poland

E-mail: eremeyev.victor@gmail.com

W. Pietraszkiewicz

Don State Technical University, Gagarina sq., 1, Rostov-on-Don, Russia 344000

E-mail: pietrasz@imp.gda.pl 
body, speed and/or relaxation of thermodynamic process, electrical field, possible chemical reactions and other factors. The contemporary linear phenomenologic model of stress-assisted thermodiffusion in solids undergoing infinitesimal deformations was formulated by $[46,49]$ and $[32,33]$. This research was partly summarized in some books, for example, by [50] and [43], where a number of analytically solved examples and many references to earlier works are given.

Within the continuum model of diffusion in addition to displacements and temperature, a new independent field appears called the species mass fraction or concentration $\mathrm{c}_{R}$. The general assumption is that $\mathrm{c}_{R}$ is small as compared with the body mass, so it does not influence the body mass distribution but may affect elastic properties, heat flux and other phenomena. The model was generalized in different directions, see, e.g., [62] and [3] for plasticity, [63] for viscoelasticity and [19] for phase transitions. Recently, the stress-assisted diffusion found applications, for example, for modelling lithium-ion batteries, see [9-11,14,17,29,30,56], where the action of electrical field plays a crucial role. It is also worth to mention similar problems related to moisture influence on thin-walled structures, i.e. environments, see, e.g., [15] and the reference therein. Another application is the hydrogen transport in metals which is important in pipelines due to so-called hydrogen embrittlement $[1,3,5,6,18,37,44,51]$. As an interesting application of the coupled mechano-diffusion problems to thin structures, we also mention the model of paintings degradation [24]. As temperature changes, diffusion may play a significant role for thin adhesive layers, see, e.g., [39,45,57].

To describe properly general thermodiffusion phenomena in shell structures, one needs to base the thermodynamic model on possibly most complete theoretical basis.

1. Thin-walled structural elements often work in the large deformation regime, requiring to account for loss of stability, non-elastic large-strain deformations, dependence on initial stress and strains, etc. Thus, one has to base the continuum shell thermodiffusion model on 3D nonlinear continuum thermodynamics supplemented by appropriate general relations accounting for influence of diffusing species.

2. The 2D model of shell thermodiffusion should result directly from 3D one by application of some 3Dto-2D reduction procedure. Well-known 2D shell models of Kirchhoff-Love or Timoshenko-Reissner (shear-deformable) type are based on application of some constraints applied to kinematical relations of 3D continuum deformation. The accuracy of such constraints is still being analysed in the literature. The most general 3D-to-2D reduction procedure, which does not require applying any kinematic constraints, uses an exact through-the-thickness integration of balance laws of 3D continuum thermomechanics as described in [12,34]. Unfortunately, nobody as yet applied this approach to formulate the resultant 2D laws of shell thermodiffusion.

3. The general 3D nonlinear laws can be formulated in either description: the Eulerian (actual), the Lagrangian (referential) or various versions of mixed ones. At the 3D level, these descriptions are interrelated by known transformation formulae. When developing the 2D mathematical model for thermodiffusion of thin-walled shell structures, the Lagrangian description is preferable, because all the laws have to be finally reduced to those valid on the 2D shell base surface. In applications, the initial undeformed geometry of the base surface is usually the only one known in advance.

Taking account of argumentation given above, in the present paper we develop the new resultant 2D model of shell thermodiffusion. Our 2D model is fully nonlinear, the 3D-to-2D reduction procedure is based on throughthe-thickness integration of 3D basic balance laws and entropy imbalance of continuum thermodiffusion, and 2D laws are given in the Lagrangian description.

The paper is organized as follows. In Sect. 2 we recall, after [60] and [59], basic integral 3D laws of continuum thermomechanics written here in the Lagrangian description. In particular, the entropy imbalance law is taken in the Clausius-Duhem form suggested already by [61]. The 3D integral balance law of mass of the single diffusing species is formulated for the general case of deformation as well. The additional energy flow due to the species transport in the body is accounted for in the form analogous to that of the heat flow, see [27]. The nonlinear thermodiffusion problem in $3 \mathrm{D}$ solids is finally governed by five integral balance laws (of mass, of linear momentum, of angular momentum, of species mass and of energy) together with the integral law of entropy imbalance.

Direct through-the-thickness integration is applied in Sect. 3 to reduce the six 3D integral laws into their corresponding resultant 2D integral laws of shell thermodiffusion. In this reduction process, the $3 \mathrm{D}$ temperature and chemical potential scalar fields are represented by their 2D mean fields defined only on the middle surface of the shell. As a result of 3D-to-2D reduction procedure, on the 2D level the resultant shell thermodiffusion problem becomes described by more thermomechanic fields than the original 3D thermodiffusion problem of the body. Then, the 2D local laws of shell thermodiffusion and corresponding 2D mechanical and thermal boundary conditions are formulated in Sect. 4 applying the surface divergence theorems. 
In Sect. 5, we discuss possible forms of 2D constitutive equations allowed by the resultant $2 \mathrm{D}$ entropy imbalance. Introducing the 2D free energy and 2D grand-canonical energy, we derive various forms of the entropy production. The analysis of constitutive relations of elastic thermodiffusive shells is given. Since the entropy production should always be nonnegative, from the Coleman-Noll procedure this results in several constitutive restrictions leading to the $2 \mathrm{D}$ constitutive equations. As a result, we derive the relations between the stress resultants and couples, resultant entropy, mean chemical potential of the species and the 2D free energy density. For thermoelastic shells with diffusion, the entropy production reduces to more simple form called the reduced entropy inequality which restricts the possible forms of the $2 \mathrm{D}$ heat, species mass and other fluxes.

\section{Basic 3D relations of continuum thermodiffusion}

Let $\mathrm{P}$ with boundary $\partial \mathrm{P}$ be a part of the fixed reference placement $\mathrm{B}$ of the material body $\mathcal{B}$ at time $t=0$. The 3D continuum thermodiffusion phenomena are governed by six integral laws, which are extensions of five integral laws of continuum thermomechanics, see, for example, [47,59,60], with additional account for the species diffusion. Since in applications time scales associated with species diffusion are usually considerably longer than those associated with wave propagation, we neglect here the kinetic energy and all inertial effects.

The kinematics of a medium is described by a mapping $\chi_{t}$ from the reference placement $\mathrm{B}$ into the actual placement $\mathrm{B}(t)$, which has the form $\mathbf{y}=\mathbf{y}(\mathbf{x}, t)$. Here $\mathbf{y}$ is the position vector of a material particle in deformed placement of the body relative to the inertial frame $\left(\mathrm{o}, \mathbf{e}_{i}\right), i=1,2,3, \mathrm{o} \in \mathcal{E}$, where $\mathcal{E}$ is the $3 \mathrm{D}$ physical space with $\mathrm{V}$ its translation vector space, whereas $\mathbf{x}$ is the position vector of the same material particle in the reference placement relative to the same inertial frame, see Fig. 1. In what follows, we also denote by $\mathrm{x}$ and $\mathrm{y}$ the position in space corresponding to $\mathbf{x}$ and $\mathbf{y}$ in the reference and actual placements, respectively.

The mechanical balance laws of mass, linear momentum and angular momentum of any part $\mathrm{P} \subset \mathrm{B}$ of the 3D body in the referential description are well known:

$$
\begin{aligned}
& \frac{\mathrm{d}}{\mathrm{d} t} \iiint_{\mathrm{P}} \rho_{R} d \mathrm{v}=0 \\
& \iiint_{\mathrm{P}} \rho_{R} \mathbf{b} d \mathrm{v}+\iint_{\partial \mathrm{P}} \mathbf{t}_{n} d \mathrm{v}=\mathbf{0}, \\
& \iiint_{\mathrm{P}} \mathbf{y} \times \rho_{R} \mathbf{b} d \mathrm{v}+\iint_{\partial \mathrm{P}} \mathbf{y} \times \mathbf{t}_{n} d \mathbf{a}=\mathbf{0} .
\end{aligned}
$$

Here $\rho_{R}$ is the referential mass (density) of the body, $\mathbf{b}$ is the body force (density) per unit mass of $\mathcal{B}, \mathbf{t}_{n}=\mathbf{P n}_{R}$ is the contact force (density) per unit area of $\partial \mathrm{P}$ with $\mathbf{P}$ the Piola stress tensor and $\mathbf{n}_{R}$ the external unit normal vector orienting $\partial \mathrm{P}$, and $\times$ is the cross product. Since mass is not created here, it follows from (1) that $\dot{\rho}_{R} \equiv 0$ everywhere within $B$, so that the balance of body mass is identically satisfied. Hereinafter, the overdot stands for the derivative with respect to $t$.

The 3D thermomechanic balance of energy and the entropy imbalance of any part $\mathrm{P} \subset \mathrm{B}$ of the material body $\mathcal{B}$ in the referential description are

$$
\begin{aligned}
\frac{\mathrm{d}}{\mathrm{d} t} \iiint_{\mathrm{P}} \rho_{R} \varepsilon_{R} \mathrm{dv}= & \iiint_{\mathrm{P}} \mathbf{P}: \operatorname{Grad} \dot{\mathbf{y}} \mathrm{dv}+\iiint_{P} \rho_{R} \mathrm{r}_{R} \mathrm{dv} \\
& -\iint_{\partial \mathrm{P}} \mathbf{q}_{R n} \cdot \mathbf{n}_{R} \mathrm{da} \\
\frac{\mathrm{d}}{\mathrm{d} t} \iiint_{\mathrm{P}} \rho_{R} \eta_{R} \mathrm{dv} \geq & \iiint_{\mathrm{P}} \rho_{R} \mathrm{k}_{R} \mathrm{dv}-\iint_{\partial \mathrm{P}} \mathbf{j}_{R n} \cdot \mathbf{n}_{R} \mathrm{da} .
\end{aligned}
$$

Here $\varepsilon_{R}, \eta_{R}, \mathrm{r}_{R}$ and $\mathrm{k}_{R}$ are the referential strain energy, entropy, heat supply and entropy supply (densities), all per unit mass of $\mathcal{B}, \mathbf{q}_{R n}$ and $\mathbf{j}_{R n}$ are the referential vectors of heat flux and entropy flux through the boundary $\partial \mathrm{P}$, and Grad is the gradient operator in the reference placement, respectively. The scalar multiplication : in the 3D tensor space means that for any $\mathbf{A}, \mathbf{B} \in \mathrm{V} \otimes \mathrm{V}, \mathbf{A}: \mathbf{B}=\operatorname{tr}\left(\mathbf{A}^{T} \mathbf{B}\right)$, where $\operatorname{tr}$ and $T$ stand for the trace and transpose of a second-order tensor, and $\otimes$ and · denote the tensor (diad) product and the scalar product of two vectors, respectively. The balance of energy (4) is an example of a conservation law. Here the heat flux 


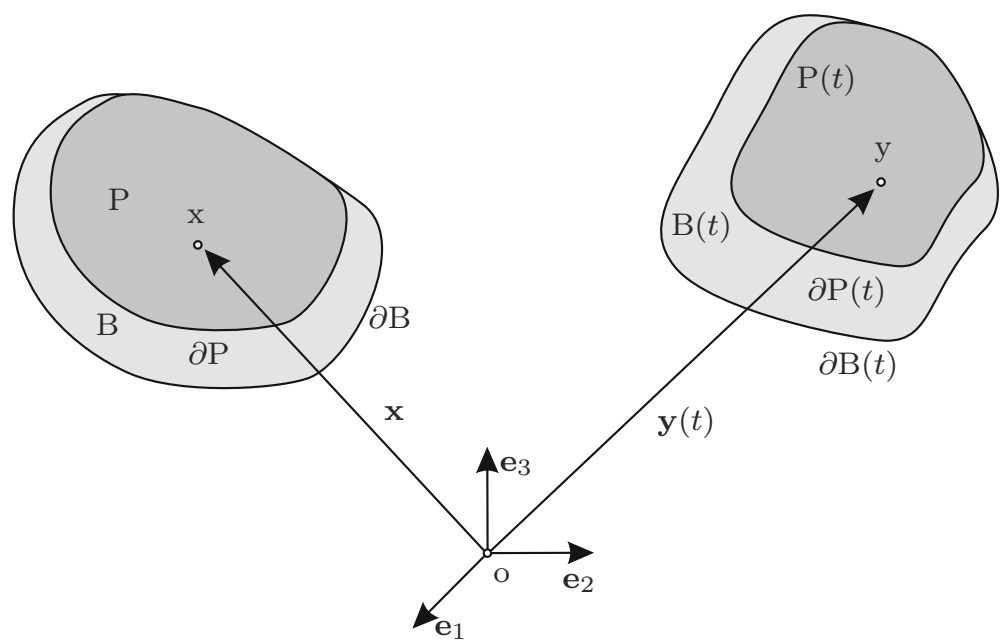

Fig. 1 Deformation: mapping form a reference placement into actual one

plays a role of the energy flux, because for simple thermoelastic media there is no other ways to transmit the non-mechanical energy, see [59].

The entropy imbalance in the form (5) is entirely decoupled from the balance laws (2), (3) and (4). In continuum thermomechanics, such coupling is achieved by introducing the absolute temperature $\theta(\mathrm{x}, t)>0$ through which the fields $\mathrm{k}_{R}$ and $\mathbf{j}_{R n}$ are related to $\mathrm{r}_{R}$ and $\mathbf{q}_{R n}$. Within rational thermomechanics used here, see [60] or [59], the coupling is assumed to be $\mathrm{k}_{R}=\mathrm{r}_{R} / \theta$ and $\mathbf{j}_{R n}=\mathbf{q}_{R n} / \theta$, so that the entropy imbalance (5) takes the Clausius-Duhem form,

$$
\frac{\mathrm{d}}{\mathrm{d} t} \iiint_{\mathrm{P}} \rho_{R} \eta_{R} \mathrm{dv} \geq \iiint_{\mathrm{P}} \rho_{R} \frac{\mathrm{r}_{R}}{\theta} \mathrm{dv}-\iint_{\partial \mathrm{P}} \frac{\mathbf{q}_{R n} \cdot \mathbf{n}_{R}}{\theta} d \mathrm{a} .
$$

Another approach to the relation between $\mathbf{j}_{R n}$ and $\mathbf{q}_{R n}$ was proposed by Ingo Müller, see [40], who introduced the extra entropy flux $\mathbf{j}_{R n}-\mathbf{q}_{R n} / \theta \neq \mathbf{0}$; see also $[35,36]$.

Let the single diffusing species be of ionic, atomic, molecular or chemical type. Let it have referential mass (density) $\rho_{R} \mathrm{c}_{R}$, where $\mathrm{c}_{R}>0$ is the species referential mass fraction. Changes in $\mathrm{c}_{R}$ in a part $\mathrm{P} \subset \mathrm{B}$ are brought about by diffusion across the boundary $\partial \mathrm{P}$ and supply in $\mathrm{P}$. These are characterized by the referential vectorial flux $\mathbf{h}_{R n}$, per unit area of $\partial \mathrm{P}$, and the referential scalar supply $\zeta_{R}$, per unit mass of $\mathrm{P}$, of the species mass, so that

$$
\iiint_{\mathrm{P}} \rho_{R} \zeta_{R} \mathrm{dv}-\iint_{\partial \mathrm{P}} \mathbf{h}_{R n} \cdot \mathbf{n}_{R} \mathrm{da}
$$

represents the net species mass entering $\mathrm{P}$ per unit time. Because $\mathbf{n}_{R}$ is the outward unit normal to $\partial \mathrm{P}$, the minus sign renders this term nonnegative when the flux points into $P$. Thus, the net rate of change of mass of the diffusing species in $\mathrm{P}$ is given by

$$
\frac{\mathrm{d}}{\mathrm{d} t} \iiint_{\mathrm{P}} \rho_{R} \mathrm{c}_{R} \mathrm{dv}=\iiint_{\mathrm{P}} \rho_{R} \zeta_{R} \mathrm{dv}-\iint_{\partial \mathrm{P}} \mathbf{h}_{R n} \cdot \mathbf{n}_{R} \mathrm{da} .
$$

This is the balance law of mass of the diffusing species.

To account for energy flow due to the species transport, several approaches were presented in the literature, see, for example, $[4,26,59,60,63]$. Here we use an approach suggested by [27] that this flow should be structurally similar to the heat flow in the body. Thus, let the energy flow due to the species transport be characterized by the chemical potential $\phi$. We assume that the species carries energy into the body by the flux $\phi \mathbf{h}_{R n}$ across the boundary $\partial \mathrm{P}$ and the supply $\rho_{R} \phi \zeta_{R}$ inside $\mathrm{P}$, so that for an arbitrary part $\mathrm{P} \subset \mathrm{B}$ the energy flow due to the species transport is

$$
\iiint_{\mathrm{P}} \rho_{R} \phi \zeta_{R} \mathrm{dv}-\iint_{\partial \mathrm{P}} \phi \mathbf{h}_{R n} \cdot \mathbf{n}_{R} \mathrm{da} .
$$

As a result, now the energy flux and supply consist of two parts: $\mathbf{q}_{R n}+\phi \mathbf{h}_{R n}$ and $\rho_{R}\left(r_{R}+\phi \zeta_{R}\right)$. Then, the $3 \mathrm{D}$ thermomechanical balance of energy (4) with account of the species energy can be refined into 


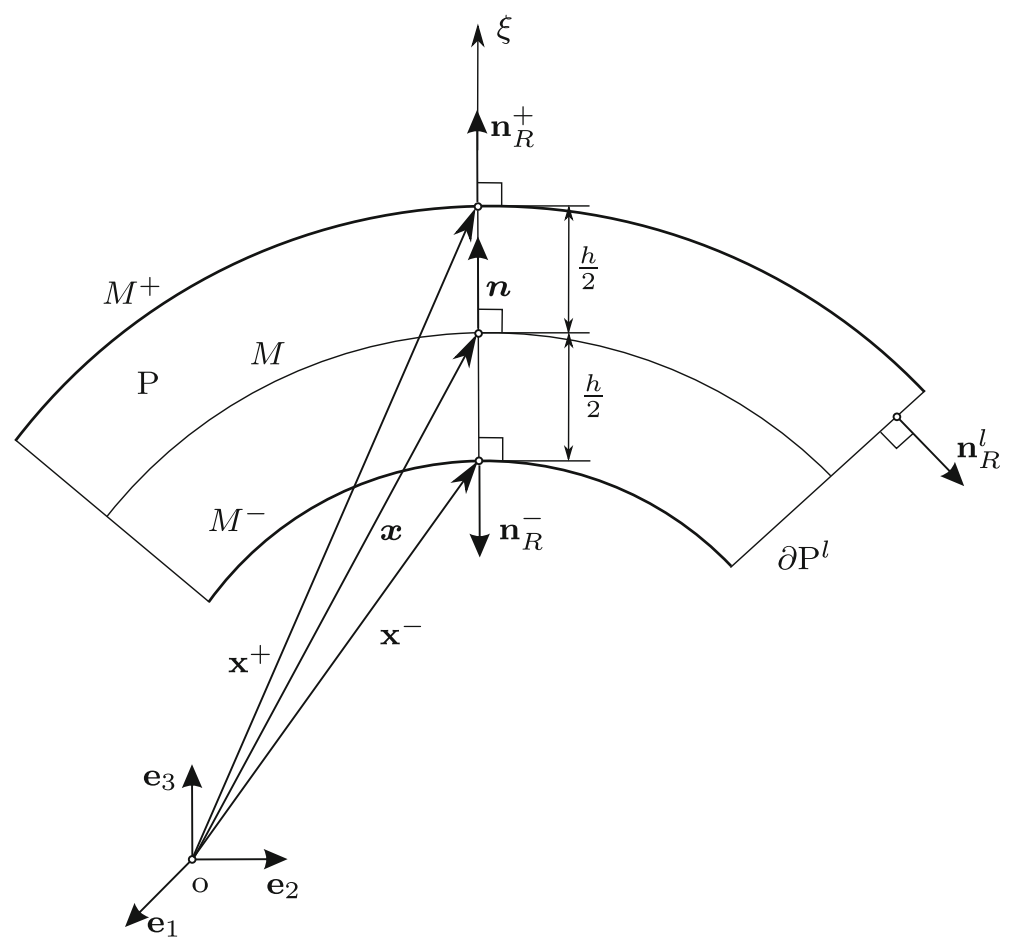

Fig. 2 Geometry of the reference shell-like region

$$
\begin{aligned}
\frac{\mathrm{d}}{\mathrm{d} t} \iiint_{\mathrm{P}} \rho_{R} \varepsilon_{R} \mathrm{dv}= & \iiint_{\mathrm{P}} \mathbf{P}: \operatorname{Grad} \dot{\mathbf{y}} \mathrm{dv}+\iiint_{\mathrm{P}} \rho_{R}\left(\mathrm{r}_{R}+\phi \zeta_{R}\right) \mathrm{dv} \\
& -\iint_{\partial \mathrm{P}}\left(\mathbf{q}_{R n}+\phi \mathbf{h}_{R n}\right) \cdot \mathbf{n}_{R} \mathrm{da} .
\end{aligned}
$$

The five global integral laws (2), (3), (6), (7) and (9) govern the 3D problem of continuum thermodiffusion with one diffusing species. Assuming that all fields are smooth in any $\mathrm{P} \subset \mathrm{B}$, it is easy to transform these integral laws into their 3D local forms by applying corresponding divergence theorems. However, for our needs here it is more convenient to reduce first the $3 \mathrm{D}$ integral laws into corresponding resultant $2 \mathrm{D}$ integral laws of shell thermodiffusion and only then to transform the 2D laws into the 2D local forms.

\section{Resultant 2D relations of shell thermodiffusion}

In the shell-like body, the boundary surface $\partial \mathrm{B}$ of the reference region consists of three parts: the upper $M^{+}$and the lower $M^{-}$shell faces, and the lateral boundary surface $\partial \mathrm{B}^{l}$ such that $\partial \mathrm{B}=M^{+} \cup M^{-} \cup \partial \mathrm{B}^{l}, M^{+} \cap M^{-}=\emptyset$. Relative to the origin $\mathrm{o} \in \mathcal{E}$ of the inertial frame the position vectors $\mathbf{x}$ of $\mathrm{B}$ and $\mathbf{y}$ of $\mathrm{B}(t)=\chi_{t}(\mathrm{~B})$ are usually described by

$$
\mathbf{x}(x, \xi)=\boldsymbol{x}(x)+\xi \boldsymbol{n}(x), \quad \mathbf{y}(x, \xi, t)=\boldsymbol{y}(x, t)+\mathbf{z}(x, \xi, t)
$$

Here $\boldsymbol{x}(x)=\mathbf{x}(x, 0)$ is the position vector of corresponding point of some reference base surface $M \subset \mathcal{E}$, $\boldsymbol{n}(x)$ is the unit normal vector orienting $M$, and $\xi \in\left[-h^{-}(x), h^{+}(x)\right]$ is the distance along $\boldsymbol{n}$ from $M$ to $\mathrm{x} \in \mathrm{B}$, with $h=h^{-}+h^{+}$the initial shell thickness. In this paper for convenience we assume that $M$ be the middle surface of $\mathrm{B}, h^{+}=h^{-}=h / 2$, with $h$ be constant everywhere, Fig. 2. Moreover, $\boldsymbol{y}(x, t)$ is the position vector of the deformed base surface $M(t)$, and $\mathbf{z}(x, \xi, t), \mathbf{z}(x, 0, t)=\mathbf{0}$, is a deviation of $\mathrm{y} \in \mathrm{B}(t)$ from the deformed base surface $M(t)$, see Fig. 3. Vectors $\mathbf{x}^{l}$ and $\mathbf{y}^{l}$ denote the position vectors of the part of the boundary where external forces $\mathbf{t}_{n}^{l}$ act, defined in the reference and actual placements, respectively. Here and in what follows we use the convention that the fields defined on the shell base surface $M$ are written by italic symbols, except in a few specially denoted cases.

Each placement of any part $\mathrm{P}(t) \subset \mathrm{B}(t)$ of the shell-like body can be represented through a part $\Pi(t)$ of the shell base surface $M(t)$ taken here to be the material surface, i.e. consisting of the same material particles 


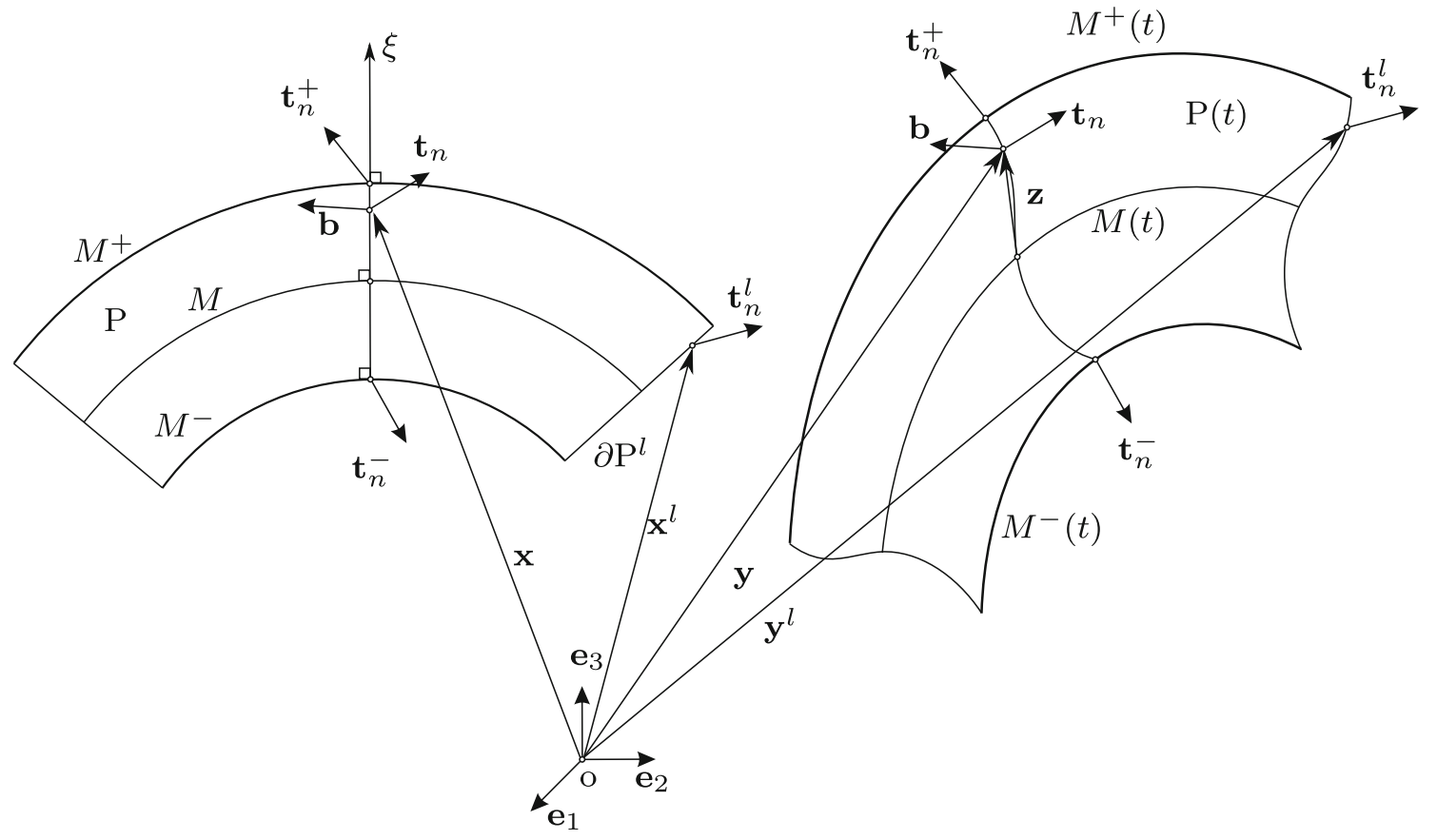

Fig. 3 Loading of the shell-like region

during the shell motion. By $y \in \Pi(t)$, we denote a point of $\Pi(t)$ and by $\boldsymbol{y}=y-\mathrm{o}$ its position vector in the inertial frame. Then, $\Pi \subset M$ represents a part of $M$, while $x \in \Pi$ is the point of $\Pi$ and $\boldsymbol{x}=x-\mathrm{o}$ its position vector in the same inertial frame.

\subsection{Resultant 2D mechanical relations}

Let us define the following resultant 2D mechanical surface fields, Fig. 3:

$$
\begin{aligned}
\rho= & \int_{-}^{+} \rho_{R} \mu d \xi, \quad \boldsymbol{n}_{v}=\int_{-}^{+} \mathbf{t}_{n} \mu d \xi, \quad \boldsymbol{m}_{v}=\int_{-}^{+} \mathbf{z} \times \mathbf{t}_{n} \mu d \xi, \\
\rho \boldsymbol{f}= & \int_{-}^{+} \rho_{R} \mathbf{b} \mu d \xi+\mathbf{t}_{n}^{+} \mu^{+}+\mathbf{t}_{n}^{-} \mu^{-}, \\
\rho \boldsymbol{c}= & \int_{-}^{+} \rho_{R} \mathbf{z} \times \mathbf{b} \mu d \xi+\mathbf{z}^{+} \times \mathbf{t}_{n}^{+} \mu^{+}+\mathbf{z}^{-} \times \mathbf{t}_{n}^{-} \mu^{-}, \\
& \int_{-}^{+} \equiv \int_{-h^{-}}^{h^{+}},
\end{aligned}
$$

where the geometric expansion factor is $\mu=\mu(\xi) \equiv 1-2 \xi H+\xi^{2} K$, with $H$ the mean curvature and $K$ the Gaussian curvature of $M$.

In (11), $\rho(x, t)>0$ is the referential resultant surface mass (density), while $\boldsymbol{f}(x, t)$ and $\boldsymbol{c}(x, t)$ are the resultant surface force and couple vectors per unit mass of $M$, respectively. Additionally, $\boldsymbol{n}_{v}(x, t)$ and $\boldsymbol{m}_{v}(x, t)$ are the surface contact-stress and couple-stress vectors describing internal mechanical interactions between the shell parts at the boundary $\partial \Pi \backslash \partial M_{f}$. By the Cauchy surface postulate, these contact stress vectors can be represented by the respective surface stress resultant $N(x, t) \in \mathrm{V} \otimes T_{x} M$ and couple-stress resultant $\boldsymbol{M}(x, t) \in \mathrm{V} \otimes T_{x} M$ tensors of the Piola type, $\boldsymbol{n}_{v}=\boldsymbol{N} \boldsymbol{v}$ and $\boldsymbol{m}_{v}=\boldsymbol{M} \boldsymbol{v}$, with $\boldsymbol{v} \in T_{x} M$ the surface unit vector externally normal to $\partial \Pi$ and $T_{x} M$ the $2 \mathrm{D}$ vector space tangent to $M$ at $x \in M$.

As a result, the global balances of momentum and moment of momentum of the shell-like body become

$$
\iint_{\Pi} \rho f \mathrm{~d} a+\int_{\partial \Pi} \boldsymbol{N} \boldsymbol{d} \mathrm{d} s=\mathbf{0}
$$




$$
\iint_{\Pi} \rho(\boldsymbol{c}+\boldsymbol{y} \times \boldsymbol{f}) \mathrm{d} a+\int_{\partial \Pi}(\boldsymbol{M} \boldsymbol{v}+\boldsymbol{y} \times \boldsymbol{N} \boldsymbol{v}) \mathrm{d} s=\mathbf{0} .
$$

Note that the 3D stress field $\mathbf{P}$ is represented here by two resultant surface fields $\boldsymbol{N}$ and $\boldsymbol{M}$.

\subsection{Reduced 2D form of entropy imbalance}

Three different 2D temperature fields appear naturally in shell thermodynamics: a reference temperature $\vartheta$ associated with the base surface, and temperatures $\theta_{-}(x, t)=\left.\theta(x, \xi, t)\right|_{\xi=-h^{-}(x)}$ and $\theta_{+}(x, t)=\left.\theta(x, \xi, t)\right|_{\xi=h^{+}(x)}$ of the lower and upper shell faces, respectively, where here $h^{+}=h^{-}=h / 2$, i.e. the undeformed base surface $M$ is the middle surface of $3 \mathrm{D}$ region $\mathrm{P}$ of the shell. Postulating some reasonable relations between the three surface temperatures, one can reduce the number of independent $2 \mathrm{D}$ temperature fields to two or to one, whichever is appropriate. Review of several such relations available in the literature is given in $[7,20,22,42,47,54,58]$, see also $[2,8,55]$ for thermoelastic beams.

In the present paper, we introduce after [47] the mean referential surface temperature $\vartheta(x, t)$ by

$$
\frac{1}{\vartheta}=\frac{1}{2}\left(\frac{1}{\theta_{-}}+\frac{1}{\theta_{+}}\right), \frac{1}{\theta(\xi)}=\frac{1}{\vartheta}+\left(\frac{1}{\theta(\xi)}-\frac{1}{\vartheta}\right), \quad \vartheta>0,
$$

where the temperatures $\theta_{ \pm}$are taken to be equal those prevailing in the adjoining external media. The decomposition $(14)_{2}$ is quite formal and does not introduce any approximation.

The boundary surface $\partial \mathrm{P}$ in (6) consists of three parts: $\partial \mathrm{P}=\left(M^{+} \cap \mathrm{P}\right) \cup\left(M^{-} \cap \mathrm{P}\right) \cup \partial \mathrm{P}^{l}$. Then, the direct through-the-thickness integration in (6) with (14) allows one to represent (6) in the form

$$
\iint_{\Pi}\left[\rho \dot{\eta}-\rho\left(\frac{r}{\vartheta}-s\right)\right] \mathrm{d} a+\int_{\partial \Pi}\left(\frac{q_{v}}{\vartheta}-s_{\nu}\right) \mathrm{d} s \geq 0
$$

where

$$
\begin{aligned}
\rho \eta & =\int_{-}^{+} \rho_{R} \eta_{R} \mu \mathrm{d} \xi \\
\rho r & =\int_{-}^{+} \rho_{R} \mathrm{r}_{R} \mu \mathrm{d} \xi-\left(\mathbf{q}_{R n}^{+} \cdot \mathbf{n}_{R}^{+} \mu^{+}+\mathbf{q}_{R n}^{-} \cdot \mathbf{n}_{R}^{-} \mu^{-}\right) \\
\rho s & =\int_{-}^{+}\left(\frac{1}{\theta(\xi)}-\frac{1}{\vartheta}\right) \rho_{R} \mathrm{r}_{R} \mu \mathrm{d} \xi+\frac{1}{2}\left(\frac{1}{\theta_{-}}-\frac{1}{\theta_{+}}\right)\left(\mathbf{q}_{R n}^{+} \cdot \mathbf{n}_{R}^{+} \mu^{+}+\mathbf{q}_{R n}^{-} \cdot \mathbf{n}_{R}^{-} \mu^{-}\right) \\
q_{v} & =\int_{-}^{+} \mathbf{q}_{R n}^{l} \cdot \mathbf{n}_{R}^{l} \mu \mathrm{d} \xi, \quad s_{v}=\int_{-}^{+}\left(\frac{1}{\theta(\xi)}-\frac{1}{\vartheta}\right) \mathbf{q}_{R n}^{l} \cdot \mathbf{n}_{R}^{l} \mu \mathrm{d} \xi
\end{aligned}
$$

Here $\eta(x, t), r(x, t)$, and $s(x, t)$ are the respective surface resultant entropy supply, heat supply and extra heat supply fields, all per unit mass of $M, \mathbf{q}_{R n}^{-}$and $\mathbf{q}_{R n}^{+}$are the given vectors of heat flux through the lower $M^{-}$ and upper $M^{+}$shell faces, respectively, $\mathbf{q}_{R n}^{l}$ is the heat flux vector through the lateral shell boundary surface $\partial \mathrm{P}^{l}$ having $\mathbf{n}_{R}^{l}$ as its external unit normal, while $q_{v}(x, t)$ and $s_{v}(x, t)$ are the resultant contact heat and extra heat fluxes through the internal boundary $\partial \Pi \subset \Pi \subset M$. By the Cauchy surface postulate, the fields $q_{\nu}$ and $s_{v}$ can be represented as $q_{v}=\boldsymbol{q} \cdot \boldsymbol{v}$ and $s_{\nu}=\boldsymbol{s} \cdot \boldsymbol{v}$, where $\boldsymbol{q}$ and $\boldsymbol{s}$ are the referential 2D resultant vectors of heat flux and extra heat flux, respectively.

\subsection{Resultant 2D species mass balance}

For the balance of species mass (7), we introduce the following resultant fields:

$$
\begin{aligned}
& \rho c=\int_{-}^{+} \rho_{R} c_{R} \mu d \xi, \quad h_{v}=\int_{-}^{+} \mathbf{h}_{R n}^{l} \cdot \mathbf{n}_{R}^{l} \mu \mathrm{d} \xi, \\
& \rho \zeta=\int_{-}^{+} \rho_{R} \zeta_{R} \mu \mathrm{d} \xi-\left(\mathbf{h}_{R n}^{+} \cdot \mathbf{n}_{R}^{+} \mu^{+}+\mathbf{h}_{R n}^{-} \cdot \mathbf{n}_{R}^{-} \mu^{-}\right),
\end{aligned}
$$


where $c(x, t)$ is the 2D resultant mass fraction of the species, per unit mass of $M, \mathbf{h}_{R n}^{-}$and $\mathbf{h}_{R n}^{+}$are given species mass flux vectors through the lower $M^{-}$and upper $M^{+}$shell faces, respectively, $\mathbf{h}_{R n}^{l}$ is the species mass flux vector through the lateral shell boundary surface $\partial \mathrm{P}^{l}, h_{v}=\boldsymbol{h} \cdot \boldsymbol{v}$ is the resultant species mass flux through $\partial \Pi$ with $\boldsymbol{h}$ the corresponding $2 \mathrm{D}$ resultant mass flux vector, per unit length of $\partial \Pi$, and $\zeta(x, t)$ is the 2D resultant species mass supply per unit mass of $\Pi$.

Now the species mass balance takes the resultant form

$$
\frac{\mathrm{d}}{\mathrm{d} t} \iint_{\Pi} \rho c \mathrm{~d} a=\iint_{\Pi} \rho \zeta \mathrm{d} a-\int_{\partial \Pi} \boldsymbol{h} \cdot \boldsymbol{v} \mathrm{d} s .
$$

\subsection{Resultant 2D energy balance}

Finally, we can introduce the resultant 2D internal energy surface field

$$
\rho \varepsilon=\int_{-}^{+} \rho_{R} \varepsilon_{R} \mu \mathrm{d} \xi
$$

In (19), $\varepsilon(x, t)$ is defined per unit mass of $M$.

Reduction to 2D form of the first 3D term $\mathbf{P}: \operatorname{Grad} \dot{\mathbf{y}} \equiv \sigma$ in the right-hand side of (9) requires a special attention. The term mechanically means the 3D stress power (density) and contains scalar multiplication of two 3D tensor fields: the Piola stress tensor $\mathbf{P}$ and the gradient of deformation rate Grad $\dot{\mathbf{y}}$. Thanks to [12,34] and [48], the 2D resultant stress power can uniquely be expressed in the following 2D coordinate-free form, see [21]:

$$
\begin{aligned}
\sigma \approx \sigma_{e} & =N: \boldsymbol{E}^{\circ}+\boldsymbol{M}: \boldsymbol{K}^{\circ}, \\
\boldsymbol{E} & =\boldsymbol{J} \mathbf{F}-\boldsymbol{Q I}, \quad \boldsymbol{K}=\boldsymbol{C} \mathbf{F}-\boldsymbol{Q B}, \\
\boldsymbol{E}^{\circ} & =\boldsymbol{Q} \frac{\mathrm{d}}{\mathrm{d} t}\left(\boldsymbol{Q}^{T} \boldsymbol{E}\right)=\operatorname{Grad}_{S} \boldsymbol{v}-\boldsymbol{\Omega} \boldsymbol{F}, \quad \boldsymbol{K}^{\circ}=\boldsymbol{Q} \frac{\mathrm{d}}{\mathrm{d} t}\left(\boldsymbol{Q}^{T} \boldsymbol{K}\right)=\operatorname{Grad}_{S} \boldsymbol{\omega} .
\end{aligned}
$$

Here the scalar product : used for two mixed tensors $\boldsymbol{A}, \boldsymbol{D} \in \mathrm{V} \otimes T_{x} M$ is also defined as $\boldsymbol{A}: \boldsymbol{D}=\operatorname{tr}\left(\boldsymbol{A}^{T} \boldsymbol{D}\right)$. In definitions of the natural surface stretch $\boldsymbol{E}(x, t)$ and bending $\boldsymbol{K}(x, t)$ tensors, $\boldsymbol{I} \in \mathrm{V} \otimes T_{x} M$ and $\boldsymbol{J} \in \mathrm{V} \otimes T_{y} M(t)$ are the inclusion operators at $x \in M$ and $y \in M(t)$, see [28], while $\boldsymbol{B} \in V \otimes T_{x} M$ and $\boldsymbol{C} \in V \otimes T_{y} M(t)$ are the structure tensors of the shell in the reference and actual placements, respectively, $\mathbf{F} \in T_{y} M(t) \otimes T_{x} M$ is the tangential surface deformation gradient such that $d y=\mathbf{F} d x, \boldsymbol{F}=\boldsymbol{J} \mathbf{F}=\operatorname{Grad}_{S} \boldsymbol{y}$, and $\operatorname{Grad}_{S}$ is the surface gradient operator in the reference geometry of $M$, see [28]. The co-rotational time derivative $(\ldots)^{\circ}$ is defined in (22) through the rotation tensor $\boldsymbol{Q}=\boldsymbol{d}_{i} \otimes \boldsymbol{t}_{i} \in \mathrm{V} \otimes \mathrm{V}, \boldsymbol{Q}^{T}=\boldsymbol{Q}^{-1}$, det $\boldsymbol{Q}=+1$, where $\boldsymbol{d}_{i}(x, t)$ and $\boldsymbol{t}_{i}(x, t), i=1,2,3$, are the orthonormal base vectors (directors) in the actual and reference placement, respectively. The expression (20) is some direct resultant representation of the 3D stress power by only 2D fields defined on $M$.

Moreover, now

$$
\boldsymbol{u}=\boldsymbol{y}-\boldsymbol{x}, \quad \boldsymbol{v}=\dot{\boldsymbol{y}}=\dot{\boldsymbol{u}}, \quad \boldsymbol{\omega}=\operatorname{ax}\left(\dot{\boldsymbol{Q}} \boldsymbol{Q}^{T}\right), \quad \boldsymbol{\Omega}=\boldsymbol{\omega} \times \mathbf{1},
$$

where $\boldsymbol{u}(x, t)$ is the surface translation vector, $\boldsymbol{v}(x, t)$ is the referential surface velocity vector, $\boldsymbol{Q}(x, t)$ is the surface rotation tensor and $\boldsymbol{\omega}$ is the referential surface angular velocity vector. The fields $\boldsymbol{u}$ (or $\boldsymbol{y}$ ) and $\boldsymbol{Q}$ are independent kinematic variables of the shell motion. In addition, $(\operatorname{ax} \mathbf{T}) \in \mathrm{V}$ means the axial vector of the skew tensor $\mathbf{T} \in \mathrm{V} \otimes \mathrm{V}, \mathbf{T}=-\mathbf{T}^{T}$, so that $\mathbf{T}=($ ax $\mathbf{T}) \times \mathbf{1}$, where $\mathbf{1} \in \mathrm{V} \otimes \mathrm{V}$ is the $3 \mathrm{D}$ identity tensor.

A reasonable reduction in two last integrals in (9) to their $2 \mathrm{D}$ representation is still the open problem. These integrals also contain multiplication of two 3D fields: the chemical potential $\phi$ by the flux vector $\mathbf{h}_{R n}$ or by the scalar supply $\zeta_{R}$, respectively. In some analogy to temperature distribution along the shell thickness, we note that three different 2D chemical potential fields could appear in shell thermodiffusion: a reference chemical potential $\varphi$ associated with the base surface $M$ and chemical potentials $\phi_{-}$and $\phi_{+}$of the lower and upper shell faces, respectively. Postulating some reasonable relations between these three chemical potentials, one can reduce the number of independent $2 \mathrm{D}$ chemical potentials to two or to one.

In the present paper, we use the mean referential $2 \mathrm{D}$ chemical potential $\varphi$ defined by

$$
\varphi=\frac{1}{2}\left(\phi_{+}+\phi_{-}\right), \quad \phi(\xi)=\varphi+(\phi(\xi)-\varphi) .
$$


Again, the decomposition $(24)_{2}$ is quite formal and does not introduce any approximation. Then, by direct through-the-thickness integration the 3D energy flow due to the species transport (8) can be reduced to its $2 \mathrm{D}$ form

$$
\iint_{\Pi} \rho(\varphi \zeta+\varsigma) \mathrm{d} a-\int_{\partial \Pi}\left(\varphi h_{\nu}+p_{\nu}\right) \mathrm{d} s,
$$

where $\rho \zeta$ and $h_{v}=\boldsymbol{h} \cdot \boldsymbol{v}$ are defined in (17), and

$$
\begin{aligned}
p_{v} & =\int_{-}^{+}(\phi(\xi)-\varphi) \mathbf{h}_{R n}^{l} \cdot \mathbf{n}_{R}^{l} \mu \mathrm{d} \xi, \\
\rho \zeta & =\int_{-}^{+}(\phi(\xi)-\varphi) \rho_{R} \zeta_{R} \mu \mathrm{d} \xi-\left(\phi_{+}-\varphi\right) \mathbf{h}_{R n}^{+} \cdot \mathbf{n}_{R}^{+} \mu^{+}-\left(\phi_{-}-\varphi\right) \mathbf{h}_{R n}^{-} \cdot \mathbf{n}_{R}^{-} \mu^{-},
\end{aligned}
$$

Here $\varsigma(x, t)$ is the extra 2D resultant species mass supply per unit mass of $\Pi$, and $p_{v}=\boldsymbol{p} \cdot \boldsymbol{v}$ is the extra 2D resultant species mass flux through $\partial \Pi$ with $\boldsymbol{p}$ the corresponding extra resultant $2 \mathrm{D}$ species mass flux vector, per unit length of $\partial \Pi$.

Taking this representation, the 2D resultant fields (19) and the 2D form (20) of the stress power, the resultant $2 \mathrm{D}$ representation of the balance of energy (9) is

$$
\begin{aligned}
\iint_{\Pi} \rho \dot{\varepsilon} \mathrm{d} a= & \iint_{\Pi}\left(\rho r+\sigma_{e}\right) \mathrm{d} a-\int_{\partial \Pi} q_{\nu} \mathrm{d} s \\
& +\iint_{\Pi} \rho(\varphi \zeta+\varsigma) \mathrm{d} a-\int_{\partial \Pi}\left(\varphi h_{\nu}+p_{\nu}\right) \mathrm{d} s .
\end{aligned}
$$

\subsection{D fields and their 2D counterparts}

Summarizing the results presented above, let us note that after the through-the-thickness integration the reduction in 3D governing balance and imbalance equations to 2D resultant ones generates additional surface fields, see Table 1 . Here in addition to the mean surface field $\vartheta(x, t)$ introduced by (14), we have the respective dual field, which is the resultant 2D surface entropy $\eta(x, t)$. This field appears naturally as multiplier of $\vartheta$ after the through-the-thickness integration of the 3D entropy distribution $\eta_{R}(x, \xi, t)$ compatible with 3D temperature distribution (14), see [47] for details.

Following [27], we have considered here the 3D chemical potential which has been introduced to characterize the energy changes due to the species mass transport. Appeared naturally is the 2D surface field of mean chemical potential $\varphi(x, t)$. The $2 \mathrm{D}$ field $\varphi(x, t)$ requires the respective dual field which is the $2 \mathrm{D}$ mean mass fraction $c(x, t)$ characterizing the non-homogeneous distribution of the 3D species mass across the thickness of the shell-like solid.

In addition to $\varepsilon$, we introduce here the surface free energy density $\psi=\psi(x, t)$ and the surface grandcanonical energy density $\omega=\omega(x, t)$ with their 3D counterparts $\psi_{R}=\varepsilon_{R}-\theta \eta_{R}$ and $\omega_{R}=\psi_{R}-\phi \mathrm{c}_{R}$ by the relations

$$
\psi=\varepsilon-\vartheta \eta, \quad \omega=\varepsilon-\vartheta \eta-\varphi c .
$$

\section{Local form of the 2D BVP}

The nonlinear problem of 2D resultant shell thermodiffusion is governed by the global balance laws of linear momentum (10), of angular momentum (11) and of diffusing species mass (12), together with the balance of energy (17) and the entropy imbalance (19).

Let us apply to these global laws the surface divergence theorems

$$
\begin{aligned}
\int_{\partial \Pi} \boldsymbol{a} \cdot \boldsymbol{v} \mathrm{d} s & =\iint_{\Pi} \operatorname{Div}_{S} \boldsymbol{a} \mathrm{d} a, \quad \int_{\partial \Pi} \boldsymbol{S} \boldsymbol{v} \mathrm{d} s=\iint_{\Pi} \operatorname{Div}_{S} \boldsymbol{S} \mathrm{d} a \\
\int_{\partial \Pi} \boldsymbol{a} \times \boldsymbol{S} \boldsymbol{v} \mathrm{d} s & =\iint_{\Pi}\left\{\boldsymbol{a} \times \operatorname{Div}_{S} \boldsymbol{S}+\operatorname{ax}\left[\boldsymbol{S}\left(\operatorname{Grad}_{S} \boldsymbol{a}\right)^{T}-\left(\operatorname{Grad}_{S} \boldsymbol{a}\right) \boldsymbol{S}^{T}\right]\right\} \mathrm{d} \boldsymbol{a}
\end{aligned}
$$


Table $13 \mathrm{D}$ fields and their 2D counterparts

\begin{tabular}{lll}
\hline & $3 \mathrm{D}$ field & 2D fields \\
\hline Placement & $\mathbf{y}(x, \xi, t)$ & $\boldsymbol{y}(x, t), \boldsymbol{Q}(x, t)$ \\
Stresses & $\mathbf{P}(x, \xi, t)$ & $\boldsymbol{N}(x, t), \boldsymbol{M}(x, t)$ \\
Temperature & $\theta(x, \xi, t)$ & $\boldsymbol{q}(x, t)$ \\
Heat flux & $\mathbf{q}_{R}(x, \xi, t)$ & $\eta(x, t), \boldsymbol{s}(x, t)$ \\
Entropy & $\eta_{R}(x, \xi, t)$ & $\rho(x, t)$ \\
Mass density & $\rho_{R}(x, \xi, t)$ & $\varepsilon(x, t)$ \\
Internal energy & $\varepsilon_{R}(x, \xi, t)$ & $\psi(x, t)$ \\
Free energy & $\psi_{R}(x, \xi, t)$ & $c(x, t)$ \\
Species mass fraction & $\mathrm{c}_{R}(x, \xi, t)$ & $\boldsymbol{h}(x, t), \boldsymbol{p}(x, t)$ \\
Species mass flux & $\mathbf{h}_{R}(x, \xi, t)$ & $\varphi(x, t)$ \\
Chemical potential & $\phi(x, \xi, t)$ & $\omega(x, t)$ \\
Grand-canonical energy & $\omega_{R}(x, \xi, t)$ & \\
\hline
\end{tabular}

valid for any $\boldsymbol{a}(x, t) \in T_{x} M$ and $\boldsymbol{S}(x, t) \in \mathrm{V} \otimes T_{x} M$, where the surface gradient and divergence operators with respect to $x \in M$ are defined as in [28]. Then, after some transformations we obtain the following local laws of $2 \mathrm{D}$ resultant shell thermodiffusion in the referential (Lagrangian) description valid in any $\Pi \subset M$ :

$$
\begin{aligned}
& \operatorname{Div}_{S} \boldsymbol{N}+\rho \boldsymbol{f}=\mathbf{0}, \quad \operatorname{Div}_{S} \boldsymbol{M}+\operatorname{ax}\left(\boldsymbol{N} \boldsymbol{F}^{T}-\boldsymbol{F} \boldsymbol{N}^{T}\right)+\rho \boldsymbol{c}=\mathbf{0}, \\
& \rho \dot{\boldsymbol{c}}+\operatorname{Div}_{S} \boldsymbol{h}-\rho \zeta=0, \\
& \rho \dot{\varepsilon}-\left(\boldsymbol{N}: \boldsymbol{E}^{\circ}+\boldsymbol{M}: \boldsymbol{K}^{\circ}\right)-\rho r+\operatorname{Div}_{S} \boldsymbol{q}-\rho(\varphi \zeta+\varsigma)+\operatorname{Div}_{S}(\varphi \boldsymbol{h}+\boldsymbol{p})=0, \\
& \rho \dot{\eta}-\rho\left(\frac{r}{\vartheta}+s\right)+\operatorname{Div}_{S}\left(\frac{\boldsymbol{q}}{\vartheta}+\boldsymbol{s}\right) \geq 0,
\end{aligned}
$$

where $\boldsymbol{F}=\operatorname{Grad}_{S} \boldsymbol{y} \in \mathrm{V} \otimes T_{x} M$ is the surface deformation gradient.

The corresponding 2D static and thermodiffusive boundary conditions are:

$$
\begin{aligned}
& \boldsymbol{u}=\boldsymbol{u}^{*}, \quad \boldsymbol{Q}=\boldsymbol{Q}^{*} \text { along } \partial M_{d}, \\
& \boldsymbol{N} \boldsymbol{v}=\boldsymbol{n}^{*}, \quad \boldsymbol{M} \boldsymbol{v}=\boldsymbol{m}^{*} \text { along } \partial M_{f}=\partial M \backslash \partial M_{d} \text {, } \\
& \vartheta=\vartheta^{*} \quad \text { along } \partial M_{t} \text {, } \\
& \boldsymbol{q} \cdot \boldsymbol{v}=q^{*}, \boldsymbol{s} \cdot \boldsymbol{v}=s^{*} \text { along } \partial M_{h}=\partial M \backslash \partial M_{t}, \\
& c=c^{*} \quad \text { along } \partial M_{\text {diff }} \text {, } \\
& \boldsymbol{h} \cdot \boldsymbol{v}=h^{*}, \boldsymbol{p} \cdot \boldsymbol{v}=p^{*} \text { along } \partial M_{m}=\partial M \backslash \partial M_{\text {diff }},
\end{aligned}
$$

where the starred quantities are prescribed and $\partial M=\partial M_{d} \cup \partial M_{f}=\partial M_{t} \cup \partial M_{f}=\partial M_{\text {diff }} \cup \partial M_{m}$. Obviously, other mixed boundary conditions are also possible. Since (31) and (32) are evolutionary equations, the proper initial conditions at $t=0$ should also be added in the case of quasistatic thermodiffusive process.

The relations (30)-(34) are 2D resultant implications of the global laws of continuum thermodiffusion, for the shell-like body represented during quasistatic motion by the material base surface $M(t)$, which in the reference placement is $M$.

In what follows, it is convenient to introduce the referential resultant 2D stress measures $\mathbf{N}(x, t)$ and $\mathbf{M}(x, t)$ with corresponding work-conjugated 2D strain measures $\mathbf{E}(x, t)$ and $\mathbf{K}(x, t)$ defined by

$$
\mathbf{N}=Q^{T} \boldsymbol{N}, \quad \mathbf{M}=\boldsymbol{Q}^{T} \boldsymbol{M}, \quad \mathbf{E}=\boldsymbol{Q}^{T} \boldsymbol{E}, \quad \mathbf{K}=\boldsymbol{Q}^{T} \boldsymbol{K} .
$$

With $\mathbf{E}$ and $\mathbf{N}, \mathbf{K}$ and $\mathbf{M}, \sigma_{e}=\mathbf{N}: \mathbf{E}+\mathbf{M}: \mathbf{K}$. Tensors $\mathbf{N}$ and $\mathbf{M}$ are the surface 2D stress measures of second Piola-Kirchhoff type of the shell.

Because by definition $\vartheta>0$, and

$$
\operatorname{Div}_{S}\left(\frac{\boldsymbol{q}}{\vartheta}\right)=\frac{1}{\vartheta} \operatorname{Div}_{S} \boldsymbol{q}-\frac{1}{\vartheta^{2}} \boldsymbol{q} \cdot \operatorname{Grad}_{S} \vartheta
$$

we rewrite (33) in the form

$$
\rho \vartheta \dot{\eta}-\rho r+\rho \vartheta s-\vartheta \operatorname{Div}_{S}\left(\frac{\boldsymbol{q}}{\vartheta}\right)+\vartheta \operatorname{Div}_{S}(s)=\rho \vartheta \dot{\eta}-\rho r-\rho \vartheta s
$$




$$
+\operatorname{Div}_{S} \boldsymbol{q}+\vartheta \operatorname{Div}_{S} \boldsymbol{s}-\frac{1}{\vartheta} \boldsymbol{q} \cdot \operatorname{Grad}_{S} \vartheta \geq 0 .
$$

Solving the 2D balance of energy (32) for $-\rho r+\operatorname{Div}_{S} \boldsymbol{q}$, we obtain

$$
-\rho r+\operatorname{Div}_{S} \boldsymbol{q}=-\rho \dot{\varepsilon}+\sigma_{e}+\rho(\varphi \zeta+\varsigma)-\operatorname{Div}_{S}(\varphi \boldsymbol{h}+\boldsymbol{p}),
$$

which introduced into the inequality above gives

$$
\begin{gathered}
\rho \vartheta \dot{\eta}+\sigma_{e}-\rho \dot{\varepsilon} \\
+\rho(\varphi \zeta+\varsigma)-\operatorname{Div}_{S}(\varphi \boldsymbol{h}+\boldsymbol{p})-\rho \vartheta s \\
+\vartheta \operatorname{Div}_{S} \boldsymbol{s}-\frac{1}{\vartheta} \boldsymbol{q} \cdot \operatorname{Grad}_{S} \vartheta \geq 0 .
\end{gathered}
$$

With (31), we can also exclude $\operatorname{Div}_{S}(\varphi \boldsymbol{h})$ here using the relation

$$
\operatorname{Div}_{S}(\varphi \boldsymbol{h})=\boldsymbol{h} \cdot \operatorname{Grad}_{S} \varphi+\varphi \operatorname{Div}_{S} \boldsymbol{h}=\boldsymbol{h} \cdot \operatorname{Grad}_{S} \varphi+\rho \varphi \zeta-\rho \varphi \dot{\boldsymbol{c}} .
$$

As a result, after some rearrangement we get the local entropy inequality in the form

$$
\begin{aligned}
\rho \vartheta \delta \equiv & \rho \vartheta \dot{\eta}+\rho \varphi \dot{\boldsymbol{c}}+\sigma_{e}-\rho \dot{\varepsilon}+\rho \varsigma-\rho \vartheta s-\operatorname{Div}_{S} \boldsymbol{p}+\vartheta \operatorname{Div}_{S} \boldsymbol{s} \\
& -\frac{1}{\vartheta} \boldsymbol{q} \cdot \boldsymbol{g}-\boldsymbol{h} \cdot \boldsymbol{k} \geq 0 .
\end{aligned}
$$

Here $\delta$ is the entropy production per unit mass of $M$, and for brevity we have denoted $\boldsymbol{g}=\operatorname{Grad}_{S} \vartheta, \boldsymbol{k}=$ $\operatorname{Grad} s \varphi$, and $\boldsymbol{g}, \boldsymbol{k} \in T_{x} M$.

Replacing $\dot{\varepsilon}$ in (35) with the formula

$$
\dot{\psi}=\dot{\varepsilon}-\dot{\vartheta} \eta-\vartheta \dot{\eta}
$$

following from $(28)_{1}$, we obtain

$$
\begin{aligned}
\rho \vartheta \delta \equiv & \rho(\varphi \dot{c}-\dot{\vartheta} \eta-\dot{\psi})+\sigma_{e}+\rho \varsigma-\rho \vartheta s-\operatorname{Div}_{S} \boldsymbol{p}+\vartheta \operatorname{Div}_{S} \boldsymbol{s} \\
& -\frac{1}{\vartheta} \boldsymbol{q} \cdot \boldsymbol{g}-\boldsymbol{h} \cdot \boldsymbol{k} \geq 0 .
\end{aligned}
$$

Differentiating $(28)_{2}$, we also obtain

$$
\dot{\omega}=\dot{\varepsilon}-\dot{\vartheta} \eta-\dot{\varphi} c-\vartheta \dot{\eta}-\varphi \dot{c},
$$

which gives the alternative formula for the local entropy production

$$
\begin{aligned}
\rho \vartheta \delta \equiv & -\rho(\dot{\vartheta} \eta+\dot{\varphi} c+\dot{\omega})+\sigma_{e}+\rho \varsigma-\rho \vartheta s-\operatorname{Div}_{S} \boldsymbol{p}+\vartheta \operatorname{Div}_{S} \boldsymbol{s} \\
& -\frac{1}{\vartheta} \boldsymbol{q} \cdot \boldsymbol{g}-\boldsymbol{h} \cdot \boldsymbol{k} \geq 0 .
\end{aligned}
$$

Let us recall that the entropy imbalance must hold for all admissible thermodynamic processes, i.e. solutions of the 2D equations (30), (31) and (32).

\section{$52 \mathrm{D}$ constitutive equations}

The structure of 2D relations of nonlinear shell thermodiffusion given in Sects. 3 and 4 differs considerably from the structure of Cauchy-type 3D rational continuum thermomechanics.

1. The kinematic structure of our resultant 2D shell theory is that of Cosserat surface [13] for which both $\boldsymbol{y}(x, t)$ and $\boldsymbol{Q}(x, t)$ are independent field variables. There are two kinematical relations (21) ) for the 2D surface stretch $\boldsymbol{E}(x, t)$ and bending $\boldsymbol{K}(x, t)$ tensors.

2. Our resultant 2D thermodiffusive laws (32) and (33) for shells contain the extra surface heat supply $s(x, t)$ and vector heat flux $s(x, t)$ fields, which are not present in the 3D Cauchy-type continuum thermo-diffusomechanics. 
3. The additional 3D species diffusion requires the additional 3D balance law of species mass (7) and corresponding modification of 3D balance law of energy (9). When integrated through the shell thickness, this gives rise the additional resultant 2D fields $\zeta(x, t), \boldsymbol{h}(x, t), \varsigma(x, t)$ and $\boldsymbol{p}(x, t)$, which modify the resultant 2D balance of energy (32) and then the entropy imbalance (36) or (37).

Our local resultant 2D balance laws (30)-(32) and the entropy imbalance (35) (or (36), or (37)) are expressed through scalar-, vector- and tensor-valued fields on $M$. Together with corresponding boundary and initial conditions, these fields describe the shell thermo-diffuso-mechanical process over the spatial domain $x \in M$ and the time domain $t \in[0, \infty)$. Different fields play different roles in the process. The fields $\Gamma(x, t) \equiv(\boldsymbol{y}, \boldsymbol{Q}, \vartheta, c)(x, t)$ constitute the basic (primary) independent variables of the 2D resultant initialboundary-value problem (IBVP) of nonlinear shell thermodiffusion. The fields $\rho, \boldsymbol{f}, \boldsymbol{c}, \zeta, r$ defined at $(x, t) \in$ $M \times[0, \infty)$ are arbitrarily assignable. The starred boundary quantities in (34) defined at $(x, t) \in \partial M \times$ $[0, \infty)$ and with appropriate initial data at $t=0$ are assumed to be given. The remaining fields $\Sigma(x, t) \equiv$ $(\psi, \eta, \mathbf{N}, \mathbf{M}, \varphi, s, \boldsymbol{q}, \varsigma, \boldsymbol{h}, \boldsymbol{p}, \boldsymbol{s})(x, t)$ have to be specified by appropriate $2 \mathrm{D}$ constitutive equations. In our case of Lagrangian description, the constitutive functions are usually formulated relative to some known (usually undeformed) reference placement $M_{0}$ of the shell base surface which is natural for some mean 2D temperature $\vartheta_{0}$ and for some resultant mass fraction $c_{0}$ of the species.

Let us recall that in 3D rational continuum thermomechanics, several principles based on experience for formulating constitutive equations were proposed in [23,61] or [60]. These are principles of determinism, local action, material frame indifference, equipresence, fading memory and other. We suppose that at least some similar requirements may be used in formulating a part of the constitutive equations of our resultant 2D shell thermodiffusion.

The principle of material frame indifference within the Lagrangian description requires the functions $\Sigma$ to depend explicitly on the 2D Lagrangian strain measures $\mathbf{E}, \mathbf{K}$ instead of the 2D displacement fields $\boldsymbol{y}, \boldsymbol{Q}$. The principle of local action and slowness of processes discussed here suggest that, in general, the constitutive fields $\Sigma$ are supposed to be influenced only by infinitesimal neighbourhood of the independent fields $\Gamma$ in space, that is

$$
\Lambda=(\mathbf{E}, \mathbf{K}, \vartheta, \boldsymbol{g}, c, \boldsymbol{l}), \quad \boldsymbol{l}=\operatorname{Grad}_{S} c .
$$

The principle of equipresence suggests that the fields $\Sigma$ should initially be assumed as functions of all independent fields, that is $\Sigma=\widehat{\Sigma}(\Lambda)$. The $2 \mathrm{D}$ constitutive equations are required to be thermodynamically admissible such that the $2 \mathrm{D}$ entropy imbalance (36) is not violated.

Taking the material time derivative of $\rho \psi(x, t)$ together with 2D balance of mass $\dot{\rho}=0$, we have

$$
-\rho \dot{\psi}=-\rho \hat{\psi}_{, \mathbf{E}}: \dot{\mathbf{E}}-\rho \hat{\psi}_{, \mathbf{K}}: \dot{\mathbf{K}}-\rho \hat{\psi}_{, \vartheta} \dot{\vartheta}-\rho \hat{\psi}_{, \boldsymbol{g}} \cdot \dot{\boldsymbol{g}}-\rho \hat{\psi}_{, c} \dot{\boldsymbol{c}}-\rho \hat{\psi}_{, \boldsymbol{l}} \cdot \boldsymbol{l},
$$

where comma denotes partial differentiation. Introducing this result into (36), we obtain

$$
\begin{aligned}
& \rho\left(\varphi-\hat{\psi}_{, c}\right) \dot{c}-\rho\left(\eta+\hat{\psi}_{, \vartheta}\right) \dot{\vartheta}+\left(\mathbf{N}-\rho \hat{\psi}_{, \mathbf{E}}\right): \dot{\mathbf{E}}+\left(\mathbf{M}-\rho \hat{\psi}_{, \mathbf{K}}\right): \dot{\mathbf{K}} \\
& \quad-\rho \hat{\psi}_{, \boldsymbol{g}} \cdot \dot{\boldsymbol{g}}+\rho S_{-}-\rho \vartheta-\rho \hat{\psi}_{, \boldsymbol{l}} \cdot \boldsymbol{l} \\
& \quad+\vartheta \operatorname{Div}_{S} \boldsymbol{s}-\operatorname{Div}_{S} \boldsymbol{p}-\frac{1}{\vartheta} \boldsymbol{q} \cdot \boldsymbol{g}-\boldsymbol{h} \cdot \boldsymbol{k} \geq 0 .
\end{aligned}
$$

Due to $\vartheta>0, \rho>0$ and linearity of (40) in material time derivatives of various fields, which themselves can take arbitrary values, the $2 \mathrm{D}$ entropy imbalance (40) is not violated only if

$$
\varphi=\hat{\psi}_{, c}, \quad \eta=-\hat{\psi}_{, \vartheta}, \quad \mathbf{N}=\rho \hat{\psi}_{, \mathbf{E}}, \quad \mathbf{M}=\rho \hat{\psi}_{, \mathbf{K}}, \quad \hat{\psi}_{, \boldsymbol{g}}=\rho \hat{\psi}_{, l}=\mathbf{0} .
$$

This indicates that $\psi=\hat{\psi}(\mathbf{E}, \mathbf{K}, \vartheta, c)$ and the constitutive equations for $\mathbf{N}, \mathbf{M}, \eta$ and $\varphi$ can be reduced to

$$
\begin{aligned}
\mathbf{N} & =\rho \hat{\psi}_{, \mathbf{E}}=\hat{\mathbf{N}}(\mathbf{E}, \mathbf{K}, \vartheta, c), \quad \mathbf{M}=\rho \hat{\psi}_{, \mathbf{K}}=\hat{\mathbf{M}}(\mathbf{E}, \mathbf{K}, \vartheta, c), \\
\eta & =-\hat{\psi}_{, \vartheta}=\hat{\eta}(\mathbf{E}, \mathbf{K}, \vartheta, c), \quad \varphi=\hat{\psi}_{, c}=\hat{\varphi}(\mathbf{E}, \mathbf{K}, \vartheta, c) .
\end{aligned}
$$

With (41), the 2D entropy imbalance (40) reduces to

$$
\rho \varsigma-\rho \vartheta s+\vartheta \operatorname{Div}_{S} \boldsymbol{s}-\operatorname{Div}_{S} \boldsymbol{p}-\frac{1}{\vartheta} \boldsymbol{q} \cdot \boldsymbol{g}-\boldsymbol{h} \cdot \boldsymbol{k} \geq 0 .
$$


Since $\psi=\varepsilon-\vartheta \eta$ by definition, also $\varepsilon=\hat{\varepsilon}(\mathbf{E}, \mathbf{K}, \vartheta, c)$. However, the constitutive functions of the remaining fields in $\Sigma(x, t)$ may still depend on all $\Lambda$, in general.

The 2D IBVP of shell thermodiffusion implied by the 2D balance laws (30)-(32), the 2D constitutive equations (42) and those of general structure for the remaining fields $s, \boldsymbol{q}, \zeta, \boldsymbol{h}, \boldsymbol{p}, \boldsymbol{s}$, complemented with appropriate boundary and initial conditions, are extremely complex and hardly readable. This is the result that our 2D IBVP for shells is direct and exact 2D implication of the corresponding IBVP of 3D nonlinear thermo-diffuso-mechanics presented in Section 2. For some narrower classes of nonlinear shell thermodiffusion problems, we may introduce additional simplifying characterization of their constitutive modelling. Below we present an example of such possible simplifying assumptions.

Let the 2D resultant heat flux vector $\boldsymbol{q}$ and the 2D resultant vector of species flux $\boldsymbol{h}$ be characterized only by the surface gradients of mean temperature $g$ and of mean chemical potential $\varphi$ according to the respective generalizations of Fourier-type and Fick-type linear relations,

$$
q=-C g+D k, \quad h=-H k+P g .
$$

Here $\boldsymbol{C}$ is the surface thermal conductivity tensor and $\boldsymbol{H}$ the surface species mobility tensor, while the surface tensors $\boldsymbol{D}$ and $\boldsymbol{P}$ can be called the thermal diffusivity tensor and the species conductivity tensor of the shell, respectively. All the material surface tensors in (45) are related to corresponding 3D tensors, if they are available, by direct through-the-thickness integration of appropriate 3D relations. Otherwise, these surface tensors should be established by experiments corresponding to the analysed $2 \mathrm{D}$ thermo-diffuso-mechanic shell problem at hand.

The fields $s, s$ are of thermal origin. Thus, we may suppose that the approximate constitutive characterization of these fields depends primarily on 2D thermal variables $\vartheta, g$, such that

$$
s=\hat{s}(\vartheta, \boldsymbol{g}), \quad s=\hat{\boldsymbol{s}}(\vartheta, \boldsymbol{g}) .
$$

Similarly, the fields $\varsigma, \boldsymbol{p}$ are of species mass origin. Thus, we may suppose that the approximate constitutive characterization of these fields depend primarily on 2D species mass fraction variables $c, \boldsymbol{l}$, such that

$$
\varsigma=\hat{\varsigma}(c, l), \quad \boldsymbol{p}=\hat{\boldsymbol{p}}(c, \boldsymbol{l}) .
$$

With (45), (46) and (47), the resulting set of 2D equations (30)-(32) of nonlinear shell thermodiffusion becomes expressed only in terms of independent field variables $\boldsymbol{y}, \boldsymbol{Q}, \vartheta$. In particular, the corresponding local resultant balance laws (31) and (32) are simplified to

$$
\begin{aligned}
& \rho \dot{c}+\operatorname{Div}_{S}(\boldsymbol{H} \boldsymbol{k}-\boldsymbol{P g})+\rho \zeta=0, \\
& \rho \dot{\varepsilon}-(\mathbf{N}: \dot{\mathbf{E}}+\mathbf{M}: \dot{\mathbf{K}})-\rho r+\operatorname{Div}_{S}(\boldsymbol{C} \boldsymbol{g}-\boldsymbol{D} \boldsymbol{k})-\rho(\varphi \zeta+\varsigma) \\
& \quad+\operatorname{Div}_{S}[\varphi(\boldsymbol{H} \boldsymbol{k}-\boldsymbol{P} \boldsymbol{g})]=0 .
\end{aligned}
$$

\section{Conclusions}

We have developed the most complete resultant 2D model of elastic shell thermodiffusion. Our 2D model is fully nonlinear and follows by direct through-the-thickness integration of 3D balance laws of mass of the body and diffusing species, linear and angular momentum, and energy as well as of entropy imbalance of continuum thermodiffusion. Our 2D model contains several additional 2D fields which are not present in the 3D parent model.

We have discussed in more detail the elastic thermodiffusive shells. The forms of most constitutive equations have been formulated discussing restrictions of the resultant entropy imbalance imposed by modified procedure of Coleman and Noll.

Our general 2D model of shell thermodiffusion allows for a number of possible simplified models. These can be obtained applying additional restrictions on nonlinearity of the process, alternative through-the-thickness distribution of temperature and chemical potential, assuming special material symmetry of the body and diffusing species, and so on. Such simplified models can be more appropriate to analyse a specific problem at hand. 
Open Access This article is licensed under a Creative Commons Attribution 4.0 International License, which permits use, sharing, adaptation, distribution and reproduction in any medium or format, as long as you give appropriate credit to the original author(s) and the source, provide a link to the Creative Commons licence, and indicate if changes were made. The images or other third party material in this article are included in the article's Creative Commons licence, unless indicated otherwise in a credit line to the material. If material is not included in the article's Creative Commons licence and your intended use is not permitted by statutory regulation or exceeds the permitted use, you will need to obtain permission directly from the copyright holder. To view a copy of this licence, visit http://creativecommons.org/licenses/by/4.0/.

\section{A 3D local relations of nonlinear thermodiffusion in solids}

Since $\mathrm{P}$ is an arbitrary part of $\mathrm{B}$, by the use of 3D divergence theorems from (2) follows the Lagrangian equilibrium equation $[59,60]$

$$
\operatorname{Div}_{R} \mathbf{P}+\rho_{R} \mathbf{b}=\mathbf{0},
$$

where $\operatorname{Div}_{R}$ is the Lagrangian 3D divergence operator in the reference placement.

Taking into account (49), from (3) we get the following constraint for the Piola stress tensor:

$$
\mathbf{P}(\operatorname{Grad} \mathbf{y})^{T}=(\operatorname{Grad} \mathbf{y}) \mathbf{P}^{T} .
$$

The mass balance of the diffusing species (7) results in

$$
\rho_{R} \dot{\mathbf{c}}_{R}=\rho_{R} \zeta_{R}-\operatorname{Div}_{R} \mathbf{h}_{R n}
$$

The global energy conservation law (9) is equivalent to

$$
\rho_{R} \dot{\varepsilon}_{R}=\mathbf{P}: \operatorname{Grad} \dot{\mathbf{y}}+\rho_{R}\left(r_{R}+\phi \zeta_{R}\right)-\operatorname{Div}_{R}\left(\mathbf{q}_{R n}+\phi \mathbf{h}_{R n}\right) .
$$

Finally, the global Clausius-Duhem inequality (6) takes the local form,

$$
\rho_{R} \dot{\eta}_{R} \geq \frac{\rho_{R} \mathrm{r}_{R}}{\theta}-\operatorname{Div}_{R} \frac{\mathbf{q}_{R n}}{\theta} .
$$

This inequality can be written as

$$
\rho_{R} \theta \dot{\eta}_{R} \geq \underline{\rho_{R} r_{R}-\operatorname{Div}_{R} \mathbf{q}_{R n}}-\theta \mathbf{q}_{R n} \cdot \operatorname{Grad} \frac{1}{\theta} .
$$

With the use of (52) and (51), the underlined term in (54) takes the form

$$
\rho_{R} \mathrm{r}_{R}-\operatorname{Div}_{R} \mathbf{q}_{R n}=\rho_{R} \dot{\varepsilon}_{R}-\mathbf{P}: \operatorname{Grad} \dot{\mathbf{y}}-\rho_{R} \phi \dot{\mathbf{c}}_{R}+\mathbf{h}_{R n} \cdot \operatorname{Grad} \phi .
$$

As a result, (54) transforms into the following inequality:

$$
\rho_{R}\left(\theta \dot{\eta}_{R}-\dot{\varepsilon}_{R}+\phi \dot{\mathbf{c}}_{R}\right)-\mathbf{P}: \operatorname{Grad} \dot{\mathbf{y}}-\mathbf{h}_{R n} \cdot \operatorname{Grad} \phi-\frac{1}{\theta} \mathbf{q}_{R n} \cdot \operatorname{Grad} \theta \geq 0 .
$$

Let us note that (56) should be fulfilled for any material body. For simple materials, the internal energy density depends on the deformation gradient only. Assuming here that the internal energy density depends in addition on the species mass fraction

$$
\varepsilon_{R}=\varepsilon_{R}\left(\operatorname{Grad} \mathbf{y}, \eta_{R}, \mathrm{c}_{R}\right)
$$

we come to the 3D constitutive relations of thermoelastic media with diffusion

$$
\mathbf{P}=\rho_{R} \frac{\partial \varepsilon_{R}}{\partial \operatorname{Grad} \mathbf{y}}, \quad \theta=\frac{\partial \varepsilon_{R}}{\partial \eta_{R}}, \quad \phi=\frac{\partial \varepsilon_{R}}{\partial \mathrm{c}_{R}} .
$$

If instead of $\varepsilon_{R}$, we introduce the free energy density $\psi_{R}=\varepsilon_{R}-\theta \eta_{R}$ as function of Grad $\mathbf{y}, \theta$, and $\mathrm{c}_{R}$, $\psi_{R}=\psi_{R}\left(\operatorname{Grad} \mathbf{y}, \theta, \mathrm{c}_{R}\right)$, we get the 3D constitutive relations in another form

$$
\mathbf{P}=\rho_{R} \frac{\partial \psi_{R}}{\partial \operatorname{Grad} \mathbf{y}}, \quad \eta_{R}=-\frac{\partial \psi_{R}}{\partial \theta}, \quad \phi=\frac{\partial \psi_{R}}{\partial \mathrm{c}_{R}} .
$$


Introducing the grand-canonical energy $\omega_{R}=\varepsilon_{R}-\theta \eta_{R}-\mathrm{c}_{R} \phi, \omega_{R}=\omega_{R}(\operatorname{Grad} \mathbf{y}, \theta, \phi)$, we have still another forms of constitutive relations

$$
\mathbf{P}=\rho_{R} \frac{\partial \omega_{R}}{\partial \operatorname{Grad} \mathbf{y}}, \quad \eta_{R}=-\frac{\partial \omega_{R}}{\partial \theta}, \quad \mathrm{c}_{R}=-\frac{\partial \omega_{R}}{\partial \phi} .
$$

For the thermo-diffuso-elastic body, the Clausius-Duhem inequality (53) transforms to the reduced inequality for the heat and mass fluxes,

$$
-\mathbf{h}_{R n} \cdot \operatorname{Grad} \phi-\frac{1}{\theta} \mathbf{q}_{R n} \cdot \operatorname{Grad} \theta \geq 0 .
$$

Note that the fluxes $\mathbf{h}_{R n}$ and $\mathbf{q}_{R n}$ depend on both gradients of $\theta$ and $\phi$, in general. The left-hand side of (60) describes dissipation in elastic media related to heat propagation and diffusion. It is the entropy production which has the standard bilinear form used in the thermodynamics of irreversible processes, see [16,31,52]. The consistent with (60) linear in gradients forms of $\mathbf{q}$ and $\mathbf{h}$ can be given by

$$
\begin{aligned}
& \mathbf{q}=-\theta \mathbf{L}_{11} \operatorname{Grad} \theta-\theta \mathbf{L}_{12} \operatorname{Grad} \phi, \\
& \mathbf{h}=-\mathbf{L}_{21} \operatorname{Grad} \theta-\mathbf{L}_{22} \operatorname{Grad} \phi,
\end{aligned}
$$

where $\mathbf{L}_{\alpha \beta}=\mathbf{L}_{\alpha \beta}(\operatorname{Grad} \mathbf{y}, \theta, \phi)$ are tensors of phenomenological coefficients which form the positive definite matrix

$$
\left(\begin{array}{ll}
\mathbf{L}_{11} & \mathbf{L}_{12} \\
\mathbf{L}_{21} & \mathbf{L}_{22}
\end{array}\right)
$$

The Onsager reciprocal relations require that $\mathbf{L}_{12}=\mathbf{L}_{21}^{T}$. In particular, for isotropic media (61) and (62) reduce to

$$
\begin{aligned}
& \mathbf{q}=-\theta \mathrm{L}_{11} \operatorname{Grad} \theta-\theta \mathrm{L}_{12} \operatorname{Grad} \phi, \\
& \mathbf{h}=-\mathrm{L}_{21} \operatorname{Grad} \theta-\mathrm{L}_{22} \operatorname{Grad} \phi,
\end{aligned}
$$

with $\mathrm{L}_{12}=\mathrm{L}_{21}$, and by the positive definiteness requirements $\mathrm{L}_{11}>0, \mathrm{~L}_{22}>0$, and $\left(\mathrm{L}_{12}+\mathrm{L}_{21}\right)^{2}<4 \mathrm{~L}_{11} \mathrm{~L}_{22}$, see $[16,52]$. Constitutive relations (63) and (64) contain both Fourier's and Fick's laws. Note that since $\phi$ depends on the strains, here we also have coupling between stresses and thermodiffusion, so one can say that the latter equations describe stress-assisted thermodiffusion.

For elastic media with the use of (57) and (51), the conservation law (52) reduces to

$$
\rho_{R} \theta \dot{\eta}_{R}=\rho_{R} \mathrm{r}_{R}-\operatorname{Div}_{R} \mathbf{q}_{R n}-\mathbf{h}_{R n} \cdot \operatorname{Grad} \phi .
$$

Equilibrium equation (49), diffusion equation (51) and the thermoconductivity equation (52) (or (65)) should be complemented by proper constitutive relations and corresponding boundary conditions. Representing the boundary $\partial \mathrm{B}$ of $\mathrm{B}$ as follows

$$
\partial \mathrm{B}=\partial \mathrm{B}_{u} \cup \partial \mathrm{B}_{f}=\partial \mathrm{B}_{\theta} \cup \partial \mathrm{B}_{q}=\partial \mathrm{B}_{n} \cup \partial \mathrm{B}_{h},
$$

the boundary conditions take the form

$$
\begin{array}{rlll}
\mathbf{u}=\mathbf{u}^{*}, & \mathrm{x} \in \partial \mathrm{B}_{u}, & \mathbf{P n}_{R}=\mathbf{t}^{*}, \quad \mathrm{x} \in \partial \mathrm{B}_{f}, \\
\theta=\theta^{*}, & \mathrm{x} \in \partial \mathrm{B}_{\theta}, & \mathbf{q}_{R n} \cdot \mathbf{n}_{R}=\mathrm{q}^{*}, & \mathrm{x} \in \partial \mathrm{B}_{q}, \\
\mathrm{c}_{R}=\mathrm{c}_{R}^{*}, & \mathrm{x} \in \partial \mathrm{B}_{n}, & \mathbf{h}_{R n} \cdot \mathbf{n}_{R}=\mathrm{h}^{*}, & \mathrm{x} \in \partial \mathrm{B}_{h},
\end{array}
$$

where $\mathbf{u}^{*}, \mathbf{t}^{*}, \theta^{*}, \mathrm{q}^{*}, \mathrm{c}_{R}^{*}$ and $\mathrm{h}^{*}$ are given functions defined on corresponding parts of the boundary. Instead of (67) and (68), one can also use boundary conditions of the third kind,

$$
\mathbf{q}_{R n} \cdot \mathbf{n}_{R}+\kappa_{\theta}\left(\theta-\theta^{*}\right)=q^{*}, \quad \mathbf{q}_{R n} \cdot \mathbf{n}_{R}+\kappa_{c}\left(\mathrm{c}_{R}-\mathrm{c}_{R}^{*}\right)=h^{*},
$$

where $\kappa_{\theta}$ and $\kappa_{c}$ are material parameters.

Since (51) and (65) are evolutionary equations, for quasistatic processes we need also the initial conditions which have the form

$$
\theta=\theta^{\circ}, \quad \mathrm{c}_{R}=\mathrm{c}_{R}^{\circ}, \quad \mathrm{x} \in \mathrm{B}, \quad t=0,
$$

where $\theta^{\circ}$ and $\mathrm{c}_{R}^{\circ}$ are initial distributions of the temperature and of the species mass fraction. 


\section{References}

1. Abdolvand, H.: Progressive modelling and experimentation of hydrogen diffusion and precipitation in anisotropic polycrystals. Int. J. Plast. 116, 39-61 (2019)

2. Altenbach, H., Bîrsan, M., Eremeyev, V.A.: On a thermodynamic theory of rods with two temperature fields. Acta Mech. 223(8), 1583-1596 (2012)

3. Anand, L.: A thermo-mechanically-coupled theory accounting for hydrogen diffusion and large elastic-viscoplastic deformations of metals. Int. J. Solids Struct. 48(6), 962-971 (2011)

4. Atkin, R.J., Craine, R.E.: Continuum theories of mixtures: basic theory and historical development. Q. J. Mech. Appl. Math. 29(2), 209-244 (1976)

5. Belyaev, A.K., Polyanskiy, V.A., Porubov, A.V.: Nonlinear dynamics of hydrogen concentration in high-strength and highentropy alloys. Contin. Mech. Thermodyn. 31(6), 1785-1794 (2019)

6. Belyaev, A.K., Polyanskiy, V.A., Yakovlev, Y.A.: Stresses in a pipeline affected by hydrogen. Acta Mech. 223(8), 1611-1619 (2012)

7. Birsan, M., Altenbach, H.: On the dynamical theory of thermoelastic simple shells. ZAMM 91(6), $443-457$ (2011)

8. Bîrsan, M., Altenbach, H.: Theory of thin thermoelastic rods made of porous materials. Arch. Appl. Mech. 81(10), 1365-1391 (2011)

9. Bower, A.F., Chason, E., Guduru, P.R., Sheldon, B.W.: A continuum model of deformation, transport and irreversible changes in atomic structure in amorphous lithium-silicon electrodes. Acta Mater. 98, 229-241 (2015)

10. Bower, A.F., Guduru, P.R., Sethuraman, V.A.: A finite strain model of stress, diffusion, plastic flow, and electrochemical reactions in a lithium-ion half-cell. J. Mech. Phys. Solids 59(4), 804-828 (2011)

11. Bucci, G., Nadimpalli, S.P., Sethuraman, V.A., Bower, A.F., Guduru, P.R.: Measurement and modeling of the mechanical and electrochemical response of amorphous si thin film electrodes during cyclic lithiation. J. Mech. Phys. Solids 62, 276-294 (2014)

12. Chróścielewski, J., Makowski, J., Pietraszkiewicz, W.: Statics and Dynamics of Multyfolded Shells. Nonlinear Theory and Finite Elelement Method (in Polish). Wydawnictwo IPPT PAN, Warszawa (2004)

13. Cosserat, E., Cosserat, F.: Théorie des corps déformables. Herman et Fils, Paris (1909)

14. Cui, Z., Gao, F., Qu, J.: A finite deformation stress-dependent chemical potential and its applications to lithium ion batteries. J. Mech. Phys. Solids 60(7), 1280-1295 (2012)

15. Dastjerdi, S., Akgöz, B., Civalek, Ö., Malikan, M., Eremeyev, V.A.: On the non-linear dynamics of torus-shaped and cylindrical shell structures. Int. J. Eng. Sci. 156, 103,371 (2020)

16. De Groot, S.R., Mazur, P.: Non-equilibrium Thermodynamics. Dover, New York (2011)

17. Di Leo, C.V., Rejovitzky, E., Anand, L.: A Cahn-Hilliard-type phase-field theory for species diffusion coupled with large elastic deformations: application to phase-separating li-ion electrode materials. J. Mech. Phys. Solids 70, 1-29 (2014)

18. Easterling, K.E., Porter, D.A., Sherif, M.: Phase Transformations in Metals and Alloys, 3rd edn. CRC Press, Boca Raton (2009)

19. Eremeev, V.: A model of phase transitions in multicomponent elastic media. Russian J. Phys. Chem. A 77(10), 1642-1644 (2003)

20. Eremeyev, V., Pietraszkiewicz, W.: Phase transitions in thermoelastic and thermoviscoelastic shells. Archives Mech. 61(1), 41-67 (2009)

21. Eremeyev, V.A., Pietraszkiewicz, W.: Local symmetry group in the general theory of elastic shells. J. Elast. 85(2), 125-152 (2006)

22. Eremeyev, V.A., Pietraszkiewicz, W.: Thermomechanics of shells undergoing phase transition. J. Mech. Phys. Solids 59(7), 1395-1412 (2011)

23. Eringen, A.C.: Mechanics of continua. Robert E. Krieger Publishing Company, Huntington, New York (1980)

24. Eumelen, Eumelen, G.J.A.M., Bosco, E., Suiker, A.S.J., van Loon, A., Iedema, P.D.: A computational model for chemomechanical degradation of historical oil paintings due to metal soap formation. J. Mech. Phys. Solids 132, 103683 (2019)

25. Fick, A.: Ueber diffusion. Annalen der Physik 170(1), 59-86 (1855)

26. Green, A.E., Naghdi, P.M.: On thermodynamics and the nature of the second law for mixtures of interacting continua. The Quarterly J. Mech. Appl. Math. 31(3), 265-293 (1978)

27. Gurtin, M.E., Fried, E., Anand, L.: The Mechanics and Thermodynamics of Continua. Cambridge University Press, Cambridge (2010)

28. Gurtin, M.E., Murdoch, A.I.: A continuum theory of elastic material surfaces. Arch. Ration. Mech. Anal. 57(4), 291-323 (1975)

29. Haftbaradaran, H., Song, J., Curtin, W.A., Gao, H.: Continuum and atomistic models of strongly coupled diffusion, stress, and solute concentration. J. Power Sources 196(1), 361-370 (2011)

30. He, Y.L., Hu, H., Song, Y.C., Guo, Z.S., Liu, C., Zhang, J.Q.: Effects of concentration-dependent elastic modulus on the diffusion of lithium ions and diffusion induced stress in layered battery electrodes. J. Power Sources 248, 517-523 (2014)

31. Kondepudi, D., Prigogine, I.: Modern Thermodynamics: From Heat Engines to Dissipative Structures. Wiley, Chichester (1998)

32. Larché, F., Cahn, J.W.: A linear theory of thermochemical equilibrium of solids under stress. Acta Metall. 21(8), 1051-1063 (1973). https://doi.org/10.1016/0001-6160(73)90021-7

33. Larché, F.C., Cahn, J.W.: The interactions of composition and stress in crystalline solids. Acta Metall. 33(3), 331-357 (1985). https://doi.org/10.1016/0001-6160(85)90077-X

34. Libai, A., Simmonds, J.G.: The Nonlinear Theory of Elastic Shells, 2nd edn. Cambridge University Press, Cambridge (1998)

35. Maugin, G.A.: The Thermomechanics of Nonlinear Irreversible Behaviors: An Introduction. World Scientific, Singapore (1999)

36. Maugin, G.A.: On canonical equations of continuum thermomechanics. Mech. Res. Commun. 33(5), 705-710 (2006) 
37. Mehrer, H.: Diffusion in Solids: Fundamentals, Methods, Materials, Diffusion-Controlled Processes, Springer Series in Solid State Science, vol. 155. Springer Science \& Business Media, Berlin (2007)

38. Mehrer, H., Stolwijk, N.A.: Heroes and highlights in the history of diffusion. Diffus. Fundam.org 11(1), 1-32 (2009)

39. Mishuris, G., Miszuris, W., Öchsner, A.: Transmission conditions for thin reactive heat-conducting interphases: general case. In: Defect and Diffusion Forum, vol. 283, pp. 521-526. Trans Tech Publications (2009)

40. Müller, I.: On the entropy inequality. Arch. Ration. Mech. Anal. 26(2), 118-141 (1967)

41. Murch, G.E.: Diffusion in Crystalline Solids. Materials science and technology. Academic Press, New York (1984)

42. Murdoch, A.I.: A thermodynamical theory of elastic material interfaces. Q. J. Mech. Appl. Math. 29(3), $245-275$ (1976)

43. Nowacki, W., Olesiak, Z.: Thermodiffusion in Solids (in Polish). PWN, Warsaw (1991)

44. Nowick, A.S., Burton, J.J. (eds.): Diffusion in Solids. Recent Developments. Materials Science and Technology. Academic Press, New York (1975)

45. Ochsner, A., Mishuris, G., Gracio, J.: A strategy for the simulation of adhesive layers. J. Adhes. Interf. 6(1), 1-6 (2005)

46. Pidstrigach, Y.S.: Differential equations for thermal diffusion in deformed solids (in Ukrainian). Dokl. Akad. Nauk Ukr. SSR 2, 169-171 (1961)

47. Pietraszkiewicz, W.: Refined resultant thermomechanics of shells. Int. J. Eng. Sci. 49(10), 1112-1124 (2011)

48. Pietraszkiewicz, W., Chróscielewski, J., Makowski, J.: On dynamically and kinematically exact theory of shells. In: Pietraszkiewicz, W., Szymczak, C. (eds.) Shell Structures: Theory and Applications, pp. 163-167. Taylor \& Francis London, Boca Raton (2005)

49. Podstrigach, Y.S., Pavlina, V.S.: Differential equations of thermodynamic processes in n-component solid solutions. Soviet Mater. Sci. 1(4), 259-264 (1966)

50. Podstrigach, Y.S., Povstenko, Y.Z.: Introduction in Mechanics of Surface Phenomena in Deformable Solids. Naukova Dumka, Kiev (1985). (in Russian)

51. Porubov, A.V., Belyaev, A.K., Polyanskiy, V.A.: Nonlinear modeling of dynamics of hydrogen concentration in alloys. Commun. Nonlinear Sci. Numer. Simul. 90, 105,402 (2020)

52. Prigogine, I.: Introduction to Thermodynamics of Irreversible Processes, 2nd edn. Wiley, New York (1961)

53. Shewmon, P.: Diffusion in Solids, 2nd edn. Springer, Cham (2016)

54. Simmonds, J.G.: The thermodynamical theory of shells: Descent from 3-dimensions without thickness expansions. In: Axelrad, E.K., Emmerling, F.A. (eds.) Flexible Shells, Theory and Applications, pp. 1-11. Springer, Berlin (1984)

55. Simmonds, J.G.: A simple nonlinear thermodynamic theory of arbitrary elastic beams. J. Elast. 81(1), 51-62 (2005)

56. Singh, A., Pal, S.: Coupled chemo-mechanical modeling of fracture in polycrystalline cathode for lithium-ion battery. Int. J. Plast. 127, 102,636 (2020)

57. Sonato, M., Piccolroaz, A., Miszuris, W., Mishuris, G.: General transmission conditions for thin elasto-plastic pressuredependent interphase between dissimilar materials. Int. J. Solids Struct. 64, 9-21 (2015)

58. Steinmann, P., Häsner, O.: On material interfaces in thermomechanical solids. Arch. Appl. Mech. 75(1), 31-41 (2005)

59. Truesdell, C.: Rational Thermodynamics, 2nd edn. Springer, New York (1984)

60. Truesdell, C., Noll, W.: The Non-linear Field Theories of Mechanics, 3rd edn. Springer, Berlin (2004)

61. Truesdell, C., Toupin, R.: The classical field theories. In: Flügge, S. (ed.) Handbuch der Physik, III(1), pp. 226-793. Springer, Berlin (1960)

62. Villani, A., Busso, E.P., Ammar, K., Forest, S., Geers, M.G.D.: A fully coupled diffusional-mechanical formulation: numerical implementation, analytical validation, and effects of plasticity on equilibrium. Arch. Appl. Mech. 84, 1647-1664 (2014)

63. Weitsman, Y.: Stress assisted diffusion in elastic and viscoelastic materials. J. Mech. Phys. Solids 35(1), 73-93 (1987)

Publisher's Note Springer Nature remains neutral with regard to jurisdictional claims in published maps and institutional affiliations. 